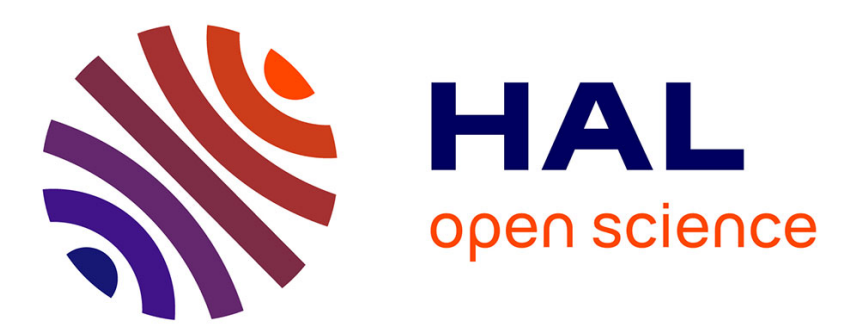

\title{
Clipping-and-Bank-Filtering Technique in Joint Crest Factor Reduction and Digital Predistortion for Power Amplifiers
}

Siqi Wang, Morgan Roger, Caroline Lelandais-Perrault

\section{- To cite this version:}

Siqi Wang, Morgan Roger, Caroline Lelandais-Perrault. Clipping-and-Bank-Filtering Technique in Joint Crest Factor Reduction and Digital Predistortion for Power Amplifiers. 2018 Asia-Pacific Microwave Conference (APMC), Nov 2018, Kyoto, Japan. 10.23919/apmc.2018.8617530 . hal01950225

\section{HAL Id: hal-01950225 \\ https://hal-centralesupelec.archives-ouvertes.fr/hal-01950225}

Submitted on 4 Jan 2019

HAL is a multi-disciplinary open access archive for the deposit and dissemination of scientific research documents, whether they are published or not. The documents may come from teaching and research institutions in France or abroad, or from public or private research centers.
L'archive ouverte pluridisciplinaire HAL, est destinée au dépôt et à la diffusion de documents scientifiques de niveau recherche, publiés ou non, émanant des établissements d'enseignement et de recherche français ou étrangers, des laboratoires publics ou privés. 


\title{
Clipping-and-Bank-Filtering Technique in Joint Crest Factor Reduction and Digital Predistortion for Power Amplifiers
}

\author{
Siqi Wang, Morgan Roger, Caroline Lelandais-Perrault \\ GeePs - Group of electrical engineering - Paris, UMR CNRS 8507, \\ CentraleSupélec, Univ. Paris-Sud, Université Paris-Saclay, Sorbonne Université \\ 3 \& 11 rue Joliot-Curie, Plateau de Moulon 91192 Gif-sur-Yvette CEDEX, France
}

\begin{abstract}
Crest factor reduction (CFR) and digital predistortion (DPD) are often used to enhance power amplifiers (PA) power efficiency while compensating for its nonlinearity. Among the joint CFR/DPDs in the literature, the one based on a parametric model has advantages on running complexity because the CFR and the DPD can be merged into a single model in the implementation. The model coefficients estimation is based on a reference correction signal computed by clipping the predistorted signal. In this paper, we propose a clippingand-bank-filtering (CABF) method for generating the reference correction signal. The performance obtained by the proposed CABF approach outperforms the conventional clipping methods in terms of adjacent channel power ratio at PA output. Its effectiveness is validated by simulation results on a Wiener model Power Amplifier with a Long Term Evolution-Advanced signal.

Index Terms-Crest factor reduction, digital predistortion, nonlinear distortion, power amplifiers, power efficiency
\end{abstract}

\section{INTRODUCTION}

The majority of power in radio frequency (RF) telecommunication systems is consumed by power amplifiers (PA). However the PA power efficiency is limited by a necessary output back-off (OBO) to prevent signal peaks going beyond the saturation point. The orthogonal frequency division multiplexing (OFDM) and recent modulation schemes impose a greater $\mathrm{OBO}$ due to their greater peak-to-average power ratio (PAPR) [1].

Digital predistortion (DPD) is widely used to compensate for the nonlinearity of the PA, which allows to make the OBO equal to the PAPR of the signal [2]. The principle of DPD is to apply a model with the inverse characteristics of the PA upstream of it [3].

The PA power efficiency can be furthermore increased by reducing the signal PAPR with different crest factor reduction (CFR) techniques [4]. Simply hard clipping (HC) the samples which exceed a given amplitude threshold will generate equally in-band and out-of-band distortion on the signal [5]. With stricter targets for the adjacent channel power ratio (ACPR), a clip-and-filter (CAF) approach is usually used to trade off in-band errors for reduced out-of-band errors [6].

There have been several studies combining CFR and DPD [5] [6] [7]. In [7], the CFR is realized with a memory polynomial (MP) model in parallel with the DPD so that the same model structure is used for the CFR and the DPD. The output of the CFR model is the correction signal, which is subtracted from the DPD output to produce a predistorted signal with reduced PAPR. Compared with conventional CFR approaches which are implemented independently in front of the DPD, this joint CFR/DPD has advantages on running complexity because the CFR and the DPD can be merged into a single model in the implementation.

The CFR model coefficients are estimated from the stimulus $u(n)$ and a reference correction signal $c_{r}(n)$ (see Fig 1). In [7], the reference correction signal is the detected peaks clipped from the predistorted signal $x(n)$ using conventional CAF. However, since $x(n)$ is not constrained in the band of the stimulus, conventional CAF cannot well reduce the out-ofband distortion at the PA output.

In this paper, we propose a clipping-and-bank-filtering $(\mathrm{CABF})$ method for generating the reference correction signal. Simulation results confirm the effectiveness of the proposed method.

\section{JOINT CFR/DPD STRUCTURE}

The architecture of the joint CFR/DPD in [7] is illustrated in Fig 1. It is based on an MP model as expressed below [3]:

$$
x_{c}(n)=\sum_{k \in \mathcal{K}} \sum_{l \in \mathcal{L}} \gamma_{k l} f_{k, l}(n)
$$

where $f_{k, l}(n)=u(n-l)|u(n-l)|^{k-1}$ are basis functions, $u(n)$ is the stimulus, $x_{c}(n)$ is the CFR/DPD output, $\gamma_{k l}$ are the model coefficients, $\mathcal{K}$ and $\mathcal{L}$ are the model nonlinearity orders and memory depths respectively.

The clipped predistorted signal is $x_{c}(n)=x(n)-c(n)$ (see Fig 1). Thus $\gamma_{k l}$ are computed as $\beta_{k l}-\alpha_{k l}$, where $\alpha$ and $\beta$ are the MP model coefficients for $c(n)$ and $x(n)$ respectively. DPD coefficients $\beta$ are estimated first according to the PA characteristics using different approaches [8]. The reference correction signal is next obtained by clipping $x(n)$ in offline processing. Then CFR coefficients $\alpha$ can be estimated to minimize the difference between $c_{r}(n)$ and $c(n)$. Consequently 


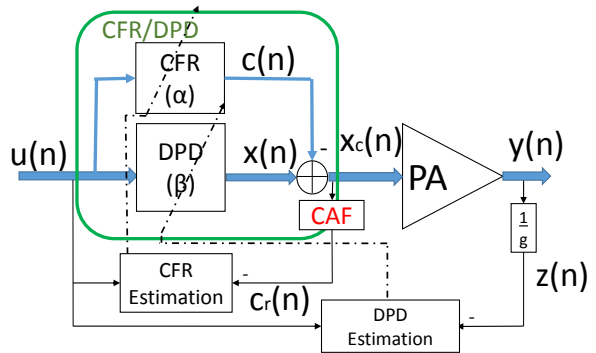

Fig. 1. Joint CFR/DPD structure in [7]

the quality of the reference correction signal $c_{r}(n)$ will have a great impact on the performance of the joint CFR/DPD.

\section{CONVENTIONAL CLIPPING METHODS}

Different methods can be used to clip $x(n)$ for $c_{r}(n)$. Here we present the conventional $\mathrm{HC}$ and $\mathrm{CAF}$.

\section{A. HC algorithm}

$\mathrm{HC}$ is one of the simplest clipping approaches. Hardclipping the signal $x(n)$ with threshold $P$, the reference correction signal can be represented as

$$
c_{r}^{h c}(n)= \begin{cases}x(n)\left(1-\frac{P}{|x(n)|}\right) & \text { if }|x(n)| \geq P \\ 0 & \text { otherwise. }\end{cases}
$$

This generates some unwanted distortions on the adjacent channels which degrade the linearization performance.

\section{B. CAF algorithm}

In order to remove the out-of-band frequency components produced by $\mathrm{HC}$, a filter that has the same bandwidth $B$ as $\boldsymbol{u}$ is applied on $c_{r}^{h c}(n)$. Since we consider here an offline CAF used to identify the CFR coefficients, an ideal rectangular window (sinc filter) can be used. The signal clipped by CAF approach is obtained in the frequency domain by

$$
\mathcal{F}\left\{c_{r}^{c a f}\right\}= \begin{cases}\mathcal{F}\left\{c_{r}^{h c}\right\}(\omega) & \text { if } \omega \in\left[-\frac{B}{2}, \frac{B}{2}\right], \\ 0 & \text { otherwise, }\end{cases}
$$

where $\mathcal{F}\{\cdot\}$ represents Fourier Transform. However new peaks in the time domain may be generated by filtering. Iterative clipand-filter approach repeats the procedure for a certain number of iterations. Since the number of peaks is reduced after each iteration, applying $\mathrm{HC}$ is tolerable in the end [6].

\section{PRoposed CABF}

Conventional CAF as above may work well when applied on the stimulus $u(n)$ since it is strictly on $\left[-\frac{B}{2}, \frac{B}{2}\right]$. However the predistorted signal $x(n)$ has out-of-band spectral components to compensate for the PA nonlinearity.

Since $x(n)$ follows an MP model same as (1), it can be decomposed with respect to different nonlinearity orders as

$$
x(n)=\sum_{k \in \mathcal{K}} g_{k}(n)
$$

where $g_{k}(n)=\sum_{l \in \mathcal{L}} \beta_{k l} f_{k, l}(n)$ is the term with nonlinearity order $k$. The spectrum of $g_{k}(n)$ depends on $k$ as it occupies the band $\left[-k \frac{B}{2}, k \frac{B}{2}\right]$. Using CAF approach (3) filters out the adjacent channel components of $g_{k}(n)$ when $k>1$. As a consequence, the performance of the conventional CAF is limited.

We propose to use a filter bank to filter each $g_{k}(n)$ according to its own band.

The algorithm comprises 4 steps:

1) Detect peaks on $x(n)$ and extract the correction signals $d_{k}(n)$ corresponding to each $g_{k}(n)$ in the decomposition (4):

$$
d_{k}(n)= \begin{cases}g_{k}(n)\left(1-\frac{P}{|x(n)|}\right) & \text { if }|x(n)| \geq P \\ 0 & \text { otherwise. }\end{cases}
$$

2) Filter $d_{k}(n)$ according to some spectral window $W_{k}(\omega)$ of width $k B$ :

$$
\mathcal{F}\left\{d_{k}^{c a f}\right\}= \begin{cases}\mathcal{F}\left\{d_{k}\right\}(\omega) \cdot W_{k}(\omega) & \text { if } \omega \in\left[-k \frac{B}{2}, k \frac{B}{2}\right], \\ 0 & \text { otherwise. }\end{cases}
$$

The clipped signal is then:

$$
x_{c}(n)=\sum_{k \in \mathcal{K}}\left(g_{k}(n)-d_{k}^{c a f}(n)\right)
$$

3) Repeat previous steps on the obtained $x_{c}(n)$ for a certain number $N$ of iterations.

4) Apply $\mathrm{HC}$ on the final $x_{c}(n)$ to ensure no peak exceeding $P$. The reference correction signal is computed as

$$
c_{r}^{c a b f}(n)=x(n)-x_{c}(n)
$$

With the proposed method above, using rectangular windows is no longer optimal. Since the band occupied by $g_{k+1}(n)$ is larger than that of $g_{k}(n)$, filtering $d_{k+1}(n)$ generates components outside $\left[-k \frac{B}{2}, k \frac{B}{2}\right]$ that can negate the effect of the filtering of $d_{k}(n)$. Consequently it is better to filter out the correction signals more on the sides of their band and less at the center so as to smoothen the resulting spectrum. A Gaussian window is used in this paper.

\section{Simulation RESUlts}

In this section, we test and compare the results of the different clipping approaches used for generating the reference correction signal: $\mathrm{HC}, \mathrm{CAF}$ and the proposed CABF.

We use a Wiener model PA for the simulation. The saturated output power of PA is $25 \mathrm{dBm}$. A $20 \mathrm{MHz}$ LTE signal with 614400 samples is used as stimulus. The number of input samples is 25000 for iterations in iterative CAF identifications. Its PAPR at the $10^{-4}$ probability level is $8 \mathrm{~dB}$.

We aim at a gain of linearized PA as its small signal gain [9], which is $21 \mathrm{~dB}$. Thus we set the input average power of DPD at $-4 \mathrm{dBm}$ to avoid the PA saturation when CFR is not applied.

The DPD model structure is chosen as

$$
\mathcal{K}=\{1,3,5,7,9\} ; \mathcal{L}=\{0,1,2,3,4,5\} .
$$




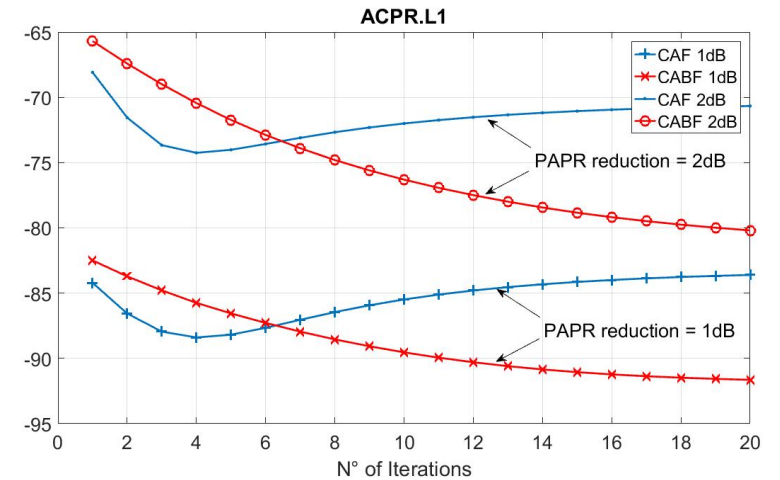

Fig. 2. Evolution of ACPR limit with the number of iterations for CAF and $\mathrm{CABF}$

TABLE I

SPECTRAL PERFORMANCES LIMITS AT THE OUTPUT OF THE PA

\begin{tabular}{|l|c|c|c|c|}
\hline \multicolumn{2}{|c|}{} & \multicolumn{2}{|c|}{ ACPR (dBc) } & EVM \\
\cline { 3 - 4 } \multicolumn{2}{|c|}{} & L1 & U1 & $(\%)$ \\
\hline PAPR & HC & -80.6 & -81.0 & 0.05 \\
\cline { 2 - 4 } $\begin{array}{l}\text { Reduction } \\
=1 \mathrm{~dB}\end{array}$ & $\mathrm{CAF}$ & -88.4 & -87.2 & 0.11 \\
\cline { 2 - 4 } & $\mathrm{CABF}$ & -90.8 & -91.6 & 0.09 \\
\hline PAPR & $\mathrm{HC}$ & -63.8 & -64.1 & 0.18 \\
\cline { 2 - 4 } $\begin{array}{l}\text { Reduction } \\
=2 \mathrm{~dB}\end{array}$ & $\mathrm{CAF}$ & -74.2 & -74.3 & 0.37 \\
\cline { 2 - 4 } & $\mathrm{CABF}$ & -80.2 & -79.3 & 0.34 \\
\hline
\end{tabular}

We feed the signal $s(n)=x(n)-c_{r}(n)$ to the PA to obtain the limits on the actual performances attainable with the clipped predistorted signal $x_{c}(n)$. These limits are independent of the model structure chosen for the CFR. We fix the DPD model coefficients so that the predistorted signal $x(n)$ is kept the same in each scenario. The performance can be evaluated with the first lower and upper channels ACPR (L1/U1) and error vector magnitude (EVM) of PA output.

As both $\mathrm{CAF}$ and $\mathrm{CABF}$ are iterative, we test their performances by varying the number $N$ of iterations from 1 to 20 as shown in Fig 2. We consider two cases of PAPR reduction: $1 \mathrm{~dB}$ and $2 \mathrm{~dB}$. In both cases, CABF outperforms CAF when $N$ is more than 6. The ACPR of CAF is not monotonously decreasing and is best for $N=4$.

The comparison of $\mathrm{HC}, \mathrm{CAF}$ and $\mathrm{CABF}$ in terms of ACPR and EVM limits is shown in Table I. The results of both CAF and CABF are for the optimal value of $N$ in the considered range, i.e. $N=4$ for CAF and $N=20$ for CABF. Compared with CAF, CABF improves both ACPR and EVM.

To compare the spectra obtained with different clipping approaches, the case of $2 \mathrm{~dB}$ PAPR reduction is illustrated in Fig 3. The proposed CABF approach (the blue curve) gives a very smooth spectrum with the lowest spectral regrowth. Consequently, it allows the joint CFR/DPD to reach better linearization performance compared with other approaches.

\section{CONCLUSION}

In this paper, we proposed a novel approach to generate the reference correction signal used to estimate the CFR model coefficients in joint CFR/DPD approach for PAPR reduction of the PA input signal. This approach is based on a

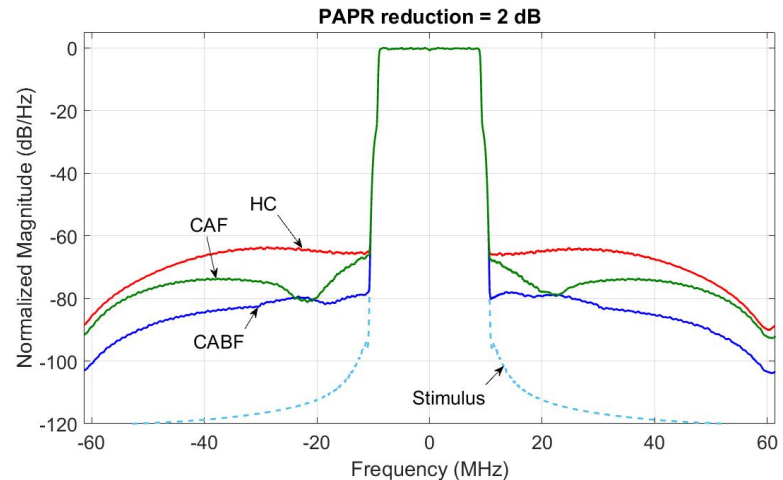

Fig. 3. Comparison of spectra at the output of the PA fed with $s(n)$ after different CFR approaches

decomposition of the predistorted signal corresponding to the different nonlinearity orders. The reference correction signal is generated by applying a filter bank of Gaussian windows. Simulations with a $20 \mathrm{MHz}$ LTE stimulus confirm that the proposed approach outperforms the conventional approaches in terms of attainable linearity of the whole system. Future work may involve looking for the best model structure for CFR/DPD and implementing the proposed method on test bench.

\section{REFERENCES}

[1] L. Guan and A. Zhu, "Green communications: Digital predistortion for wideband rf power amplifiers," IEEE Microwave Magazine, vol. 15, no. 7, pp. 84-99, Nov 2014.

[2] A. Katz, J. Wood, and D. Chokola, "The evolution of pa linearization: From classic feedforward and feedback through analog and digital predistortion," IEEE Microwave Magazine, vol. 17, no. 2, pp. 32-40, Feb 2016.

[3] J. Kim and K. Konstantinou, "Digital predistortion of wideband signals based on power amplifier model with memory," Electronics Letters, vol. 37, no. 23, pp. 1417-1418, Nov 2001.

[4] F. Sandoval, G. Poitau, and F. Gagnon, "Hybrid peak-to-average power ratio reduction techniques: Review and performance comparison," IEEE Access, vol. 5, pp. 27 145-27 161, 2017.

[5] J. Wood, Behavioral Modeling and Linearization of RF Power Amplifiers:, ser. Artech House Microwave Library. Artech House, 2014. [Online]. Available: https://books.google.fr/books?id=abpTBAAAQBAJ

[6] H. Enzinger, K. Freiberger, and C. Vogel, "Competitive linearity for envelope tracking: Dual-band crest factor reduction and 2d-vector-switched digital predistortion," IEEE Microw. Mag., vol. 19, no. 1, pp. 69-77, Jan 2018.

[7] R. N. Braithwaite, "A combined approach to digital predistortion and crest factor reduction for the linearization of an rf power amplifier," IEEE Trans. Microw. Theory Techn., vol. 61, no. 1, pp. 291-302, Jan 2013.

[8] M. Abi Hussein, V. Bohara, and O. Venard, "On the system level convergence of ila and dla for digital predistortion," in Wireless Communication Systems (ISWCS), 2012 International Symposium on, Aug 2012, pp. 870874

[9] S. Wang, M. A. Hussein, O. Venard, and G. Baudoin, "Impact of the normalization gain of digital predistortion on linearization performance and power added efficiency of the linearized power amplifier," in 2017 12th European Microwave Integrated Circuits Conference (EuMIC), Oct 2017, pp. 310-313. 